\title{
Effects of size polydispersity on random close-packed configurations of spherical particles
}

\author{
Patrick Mutabaruka $\odot,{ }^{1,2, *}$ Mahdi Taiebat $\odot,{ }^{3,1, \dagger}$ Roland J.-M. Pellenq, ${ }^{1, \$}$ and Farhang Radjai $\odot^{2,1, \S}$ \\ ${ }^{1}\langle M S E\rangle^{2}$, MIT-CNRS Joint Laboratory, Massachusetts Institute of Technology, Cambridge, Massachusetts 02139, USA \\ ${ }^{2}$ LMGC, UMR5508, CNRS-University of Montpellier, 34090 Montpellier, France \\ ${ }^{3}$ Department of Civil Engineering, University of British Columbia, Vancouver, BC V6T 1Z4, Canada
}

(Received 30 June 2019; published 11 October 2019)

\begin{abstract}
We analyze the packing properties of simulated three-dimensional polydisperse samples of spherical particles assembled by mechanical compaction with zero interparticle friction, leading to random close-packed configurations of the highest packing fraction. The particle size distributions are generated from the incomplete beta distribution with three parameters: A size span and two shape parameters that control the curvature of the distribution function. For each size distribution, the number of particles is determined by accounting for the statistical representativity of all particle size classes in terms of both the numbers and volumes of particles. Remarkably, the packing fraction increases, up to a small variability, with an effective size span, known as the coefficient of uniformity, that combines the three control parameters of the distribution. The local particle environments are characterized by the particle connectivities and anisotropies, which unveil the class of particles with four contact neighbors as the largest class with an increasing population as a function of size span, indicating the higher stability of particles trapped by four larger particles. As a result of increasing topological inhomogeneity of the packings, the force distributions get increasingly broader with increasing effective size span. Finally, we find that larger particles do not always carry stronger average stresses, in particular when the particle size distribution allows for a sufficiently large number of small particles.
\end{abstract}

DOI: 10.1103/PhysRevE.100.042906

\section{INTRODUCTION}

The hard-sphere packings have been a subject of particular interest in mathematics and physics and as models of granular materials in soil mechanics and powder technology [1-4]. Under compressive mechanical stress, spherical particles of the same size do not spontaneously get jammed in the most compact configurations, which are face-centered cubic and hexagonal close packing structures with a packing fraction $\Phi=V_{p} / V \simeq 0.74$, where $V_{p}$ and $V$ are the volume of particles and the total volume, respectively [5]. The lower packing fractions reached by compaction or other procedures are attributed to disorder in the sense of particle-positional randomness, and the resulting packings are known as random close-packed (RCP) structures with the highest packing fraction $\Phi_{c} \simeq$ 0.64 [6].

The RCP concept was revisited by Torquato et al. [7], who introduced a mathematically consistent definition of these states as maximally random jammed states depending on the order metrics and assembling protocols. In particular, this definition includes the contact-orientational (in addition to positional) randomness whose maximum value occurs in the case of the isotropic contact network. In mechanical experiments or simulations, the relevant assembling procedure is isotropic compaction under constant pressure $P$ with zero

\footnotetext{
*pmutabar@mit.edu

†mtaiebat@civil.ubc.ca

‡pellenq@mit.edu

§franck.radjai@umontpellier.fr
}

friction between particles [8]. In the absence of friction, the particle rearrangements lead to a well-defined (independent of the initial state) isotropic equilibrium state with a minimum value of the energy function $\Gamma=P V$ and hence the highest packing fraction $\Phi_{c}$ allowed by the disorder. As all normal contact forces are balanced in this state for all particles and the packing is isostatic, the coordination number is $Z=6$ (by disregarding the rattlers) [9]. This configuration is random due to the absence of long-range ordering. There is, however, no formal proof that the resulting randomness is maximal because of the constraints imposed by force balance and isostaticity. Hence, this state may also be described as a "random maximally close-packed state" and we will continue to refer to it as RCP state.

There is no reason to restrict the RCP state to monodisperse sphere packings. Broad particle size distributions (PSD) are very common in nature and industrial applications of granular materials, and they are crucial for the space-filling and strength properties of granular materials [1,3,4,10-13]. Nevertheless, packing structures have been mostly investigated in bidisperse systems, i.e., composed of two different sizes, in weakly polydisperse systems $[14,15]$, defined as systems with small size span, or in ideal apollonian or random Apollonian constructions characterized by the assumption that there is no lower bound on particle size $[4,10,16]$.

Several geometrical algorithms have been proposed to generate jammed packings of particles [4,11,12,17-20]. But to obtain RCP states by mechanical compaction, the discrete element method (DEM) is the relevant approach. The problem is that broad PSD's require a large number of particles that are not numerically tractable. Hence, the investigation of 
polydisperse granular materials requires an optimized number of particles so that all particle size classes and their volumes can be well represented. This statistical representativity of particle size classes is a function of both the size span and shape of the PSD [20]. The DEM simulations in twodimensional (2D) disk packings and 3D sphere packings with broad size spans have shown that the effective friction coefficient of granular flows is independent of PSD [13,21,22]. Furthermore, Estrada recently showed that disk packings assembled with zero friction and a power-law cumulate volume distribution (CVD) of exponent 0.5 lead to the highest packing fraction in the RCP state for a given value of the size span [23].

In this paper, we investigate the packing properties of frictionless RCP packings of spherical particles using DEM simulations for CVD's generated from the incomplete $\beta$ distribution, as in 2D studies of Voivret et al. [20]. This CVD has three parameters: Size span and two shape parameters. A pending issue that we address in this paper is how the packing fraction depends on the CVD shape parameters and coefficient of uniformity, which is a well-known parameter measured in soil mechanics to characterize the CVD. We also analyze particle connectivities and stress transmission. As we shall see, while the coordination number is weakly dependent on the CVD due to the quasi-isostaticity of the packings, the proportions of particles of different sizes with a given number of contact neighbors are sensitive to the CVD parameters.

In the following, we first introduce the numerical procedures used to generate the CVD's and sphere packings. Then we focus on the influence of the PSD parameters on the packing fraction, particle connectivities, and force distributions. We conclude with the salient results of this work and future directions of research on polydisperse granular materials.

\section{NUMERICAL PROCEDURES}

\section{A. Particle size distributions}

We assume that the particle diameters are randomly distributed between $d_{\min }$ and $d_{\max }$. The size span is characterized by the ratio

$$
\alpha=\frac{d_{\max }}{d_{\min }} .
$$

The particle diameters can be rescaled over this range:

$$
d_{r}=\frac{d-d_{\min }}{d_{\max }-d_{\min }}=\frac{d / d_{\min }-1}{\alpha-1} .
$$

This reduced size $d_{r}$ varies from 0 to 1 . The PSD over this interval can be equivalently described by one of the following forms: (1) probability distribution $P(d)$ of particle diameters $d$, (2) its cumulative form $F(d)=\int_{d_{\min }}^{d} P(x) d x$, (3) probability distribution of particle volumes $P_{v}(d)$, and (4) its cumulative form $h(d)=\int_{d_{\min }}^{d} P_{v}(x) d x$. The latter represents the cumulate volume distribution (CVD), which corresponds to the mass fraction of particles passing a sieve of mesh diameter $d$ and usually called grading curve measured in soil mechanics to describe different soil types [24]. We will use here the CVD for its clear experimental meaning and its relevance, as we shall see below. It can be alternatively written in the

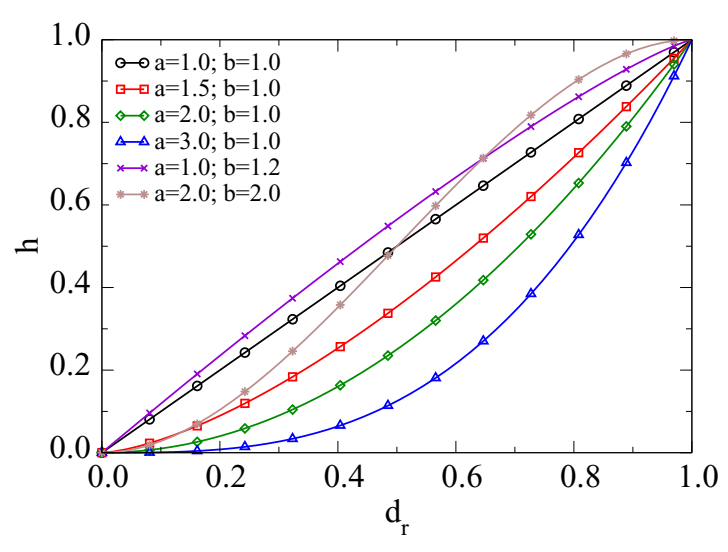

FIG. 1. Cumulate volume distributions of reduced diameters $d_{r}$ for several values of the shape parameters $a$ and $b$ considered in this paper.

following form:

$$
h(d)=\frac{\int_{d_{\min }}^{d} V(x) P(x) d x}{\int_{d_{\min }}^{d_{\max }} V(x) P(x) d x},
$$

where $V(x)$ is the volume of a particle of diameter $x$.

As in Ref. [20], we use in this work the incomplete cumulative $\beta$ distribution to model the CVD:

$$
\beta(x)=\frac{1}{B(a, b)} \int_{0}^{x} t^{a}(1-t)^{b-1} d t,
$$

where $a>0$ and $b>0$ are the two parameters of the distribution and

$$
B(a, b)=\Gamma(a) \Gamma(b) / \Gamma(a+b),
$$

where $\Gamma$ is the Gamma function. This distribution is defined and normalized over the interval $[0,1]$, so that $\beta(0)=0$ and $\beta(1)=1$. The CVD is defined by

$$
h(d)=\beta\left[d_{r}(d) ; a, b\right] .
$$

With this definition of the CVD, the span and shapes of the size distributions are controlled by three parameters: $\alpha, a$, and $b$.

Figure 1 displays the graphs of the distributions and their parameter values that will be considered below. The case $a=$ $b=1$ is a uniform distribution by volume fractions (straight line in Fig. 1). All size classes have the same volume, so that $h=d_{r}$. The CVD is double curved if $a>1$ and $b>1$. A power-law distribution is obtained if either $a$ or $b$ is set to 1 . If $a>1$ and $b=1$, then the CVD is convex, whereas for $a=1$ and $b>1$ the CVD is concave. The special case of $a=3$ and $b=1$ is a uniform distribution in particle diameters. For large values of $a$ and $b$, the distribution becomes nearly monodisperse. For $a<1$ and $b<1$, the distribution is nearly bidisperse with increasing concentration of particles around $d_{\max }$ and $d_{\min }$ for decreasing values of $a$ and $b$.

For DEM simulations, the CVD must be discretized by dividing the range $[0,1]$ of reduced diameters into $N_{c}$ size classes. Each class $i$ contains a number $N_{p / c}(i)$ of particles and has a volume $V_{p}(i)$. The total number of particles is $N_{p}=$ $\sum_{i} N_{p / c}(i)$ and the total particle volume is $V_{p}=\sum_{i} V_{p}(i)$. 


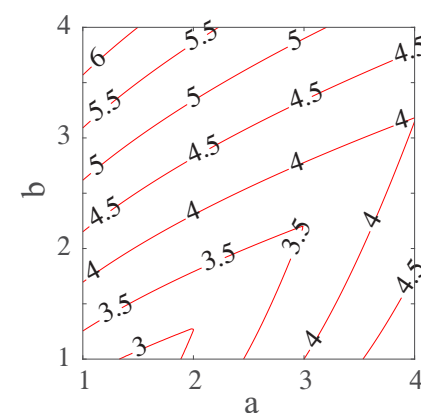

(a)

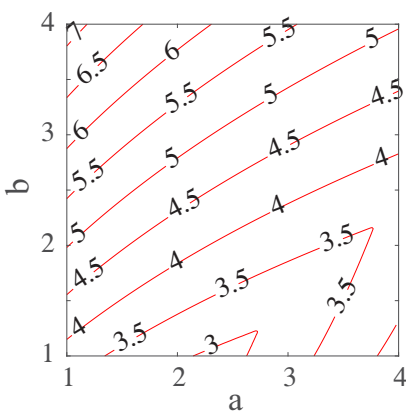

(c)

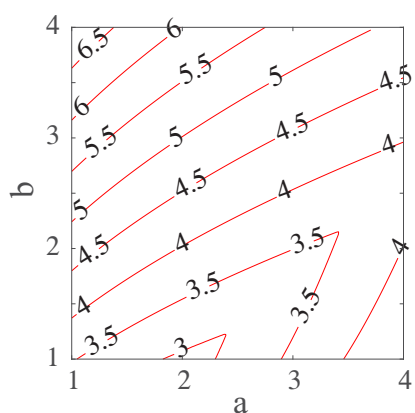

(b)

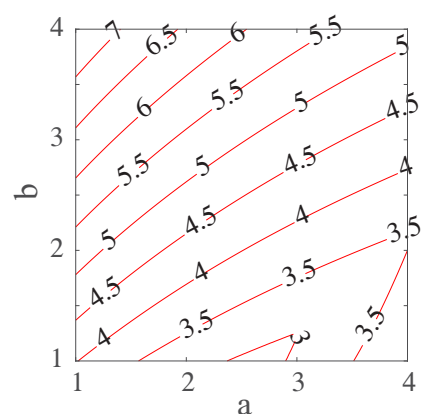

(d)
FIG. 2. Domains of statistically accessible shape parameters $a$ and $b$ for various size ratios of (a) $\alpha=2$, (b) $\alpha=3$, (c) $\alpha=4$, and (d) $\alpha=5$ for $N_{c}=10$ and $N_{p / c}^{\min }=50$. The numbers on the contour lines show $\log _{10}\left(N_{p}^{\min }\right)$, where $N_{p}^{\min }$ is the minimum number of particles needed for the statistical representativity of samples generated according to a size distribution.

Since the system is polydisperse, for a given number $N_{p}$ of particles, the number $N_{c}$ of size classes and the values of $N_{p / c}(i)$ must be such that in each class both particle volumes and their numbers are correctly represented. This requires a sufficiently large number $N_{p / c}^{\min }$ of largest particles and a sufficiently large volume $V_{p}^{\min }$ of smallest particles. If these two conditions are satisfied, then both the numbers and volumes of particles in all size classes will lie within the prescribed range, i.e., $N_{p / c}^{\min }<N_{p / c}(i)$ and $V_{p}^{\min }<V_{p}(i), \forall i$. The values of $N_{p / c}^{\min }$ and $V_{p}^{\mathrm{min}}$ depend on the number $N_{c}$ of size classes. For a given distribution $h(d)$, they determine also the minimum number $N_{p}^{\min }$ of particles needed for the representativity of the samples and it should be set according to the computation power and memory.

To give an idea of the necessary number of particles to achieve statistically representative discrete distributions of particle sizes, we calculated the number of particles $N_{p}^{\min }$ by setting $N_{p / c}^{\min }=50, N_{c}=10$, and $V_{p}^{\min }=N_{p / c}^{\min } V_{p}\left(d_{\max }\right)$. For our spherical particles, we have $V_{p}\left(d_{\max }\right)=\pi d_{\max }^{3} / 6$. Figure 2 shows the isovalues of $N_{p}^{\min }$ for several values $\alpha=[2,3,4,5]$ that were used to construct our samples. The numbers on the contour lines show $\log _{10}\left(N_{p}^{\min }\right)$. We see that the minimum number of particles is a rapidly increasing function of $\alpha$ but also of shape parameters $a$ and $b$. These data show that with a total number of particles $N_{p}=25000$ and $N_{c}=10$, it is possible to vary $\alpha$ in the range [1,5] and the shape parameters in the range $[1,3]$.
TABLE I. Simulated samples.

\begin{tabular}{lccccc}
\hline \hline ID & Dispersity & $\alpha$ & $a$ & $b$ & $N_{p}^{\text {mina }}$ \\
\hline S1 & Mono & 1 & - & - & - \\
S2 & Poly & 2 & 1 & 1 & 1581 \\
S3 & Poly & 3 & 1 & 1 & 3467 \\
S4 & Poly & 4 & 1 & 1 & 6376 \\
S5 & Poly & 5 & 1 & 1 & 10548 \\
S6 & Poly & 5 & 1.5 & 1 & 3581 \\
S7 & Poly & 5 & 2 & 1 & 1592 \\
S8 & Poly & 5 & 3 & 1 & 1200 \\
S9 & Poly & 5 & 1 & 1.2 & 19355 \\
S10 & Poly & 5 & 2 & 2 & 21707 \\
S21 & Poly & 2 & 3 & 1 & 10001 \\
S31 & Poly & 3 & 3 & 1 & 3902 \\
S41 & Poly & 4 & 3 & 1 & 2006 \\
\hline \hline
\end{tabular}

${ }^{a}$ Minimum number of particles needed for satisfying $N_{p / c}^{\min }=50$ and $N_{c}=10$.

The choice of 10 size classes $\left(N_{c}=10\right)$ is motivated by the observation that for lower number of classes the number of particles of the same size in each class will increase, and thus clustered domains of particles of the same size may occur inside the packing. This is not desirable as the "typical" local environments should be composed of particles of different sizes (and not particles of the same size). Another method that can be used for a smaller number of classes consists of introducing size variability of the particles in each class. But such an intraclass distribution can override the general interclass size distribution when large populations of particles are considered in each class. For this reason, it seems more reasonable to increase the number of size classes at the expense of decreasing the numbers of particles in each class.

The choice of $N_{p / c}^{\min }=50$ was guided, on one hand, by the fact that for $\alpha=5$ the ratio of the volumes between the largest and smallest particles is quite high $\left(5^{3}\right)$ so that larger values of $N_{p / c}^{\min }$ would require a much higher number of smaller particles and thus a much larger number of all particles. On the other hand, the local environments (first neighboring shells) of 50 large particles leads to a statistically fair estimation of the packing state as a whole. All the simulated combinations of $a, b$, and $\alpha$ used in our simulations are given in Table I and identified with a symbol that will be used to refer to our samples below. The table includes also a monodisperse system that we simulated for the sake of comparison with the polydisperse systems.

\section{B. Sample preparation protocol}

Once the particles with their sizes are generated, they are placed at random positions inside a cell and subjected

TABLE II. DEM parameters.

\begin{tabular}{lc}
\hline \hline Description & Value \\
\hline Normal contact stiffness & $10^{6} \mathrm{~N} / \mathrm{m}$ \\
Normal damping coefficient & $1500 \mathrm{~kg} / \mathrm{ms}$ \\
Density & $2500 \mathrm{~kg} / \mathrm{m}^{3}$ \\
\hline \hline
\end{tabular}




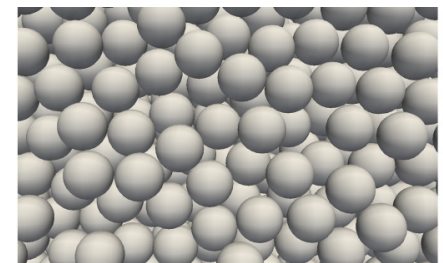

(a)

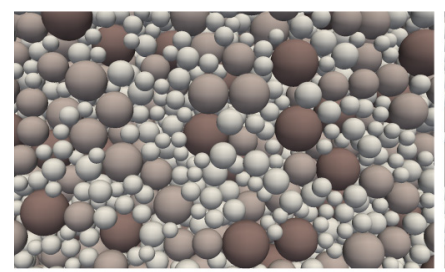

(c)

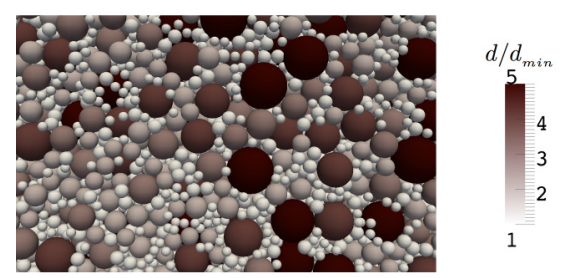

(e)

FIG. 3. Snapshots of the packings S1-S5 (a)-(e), corresponding to $\alpha=[1,2,3,4,5]$. Color intensities are proportional to the relative particle size $d / d_{\min }$.

to uniaxial compression by applying a constant pressure $\sigma_{z z}$ on the top wall, the bottom wall being frozen, and using periodic boundary conditions along the $x$ and $y$ directions. The particles are given a zero coefficient of friction $(\mu=0)$ with other particles and with the top and bottom walls. In our DEM algorithm, we use a linear viscoelastic contact law. Hence, the energy is dissipated only by normal viscous damping between particles and with the top and bottom walls. The compaction is fairly fast at the beginning but considerably slows down as the RCP state is approached. During compaction, we monitor the total kinetic energy, the contact network, and the packing fraction $\Phi$. When the kinetic energy is a small fraction of $\sigma_{z z} V$, which represents the potential energy due to the applied load, and the packing fraction and contact network are stable, we stop the simulation.

The simulation parameters are given in Table II. An important parameter is the maximum contact deflection $\delta_{\max }$ (overlap between particles). We set the applied pressure $\sigma_{z z}$ to a value such that $\delta_{\max } \sim 10^{-3} d_{\max }$. This means that the particles can be considered as nearly undeformable, and the calculated values of the packing fraction are not influenced by contact deflections. Figure 3 displays several zooms on the RCP packings obtained for $\alpha=[1,2,3,4,5]$ with $a=b=1$.

\section{PACKING FRACTION}

The packing fraction $\Phi$ is calculated by dividing the total volume of the particles $V_{p}$ by the volume of the simulation cell $V$. Figure 4 shows $\Phi$ as a function of size ratio $\alpha$ for all our particle size distributions listed in Table I. In agreement

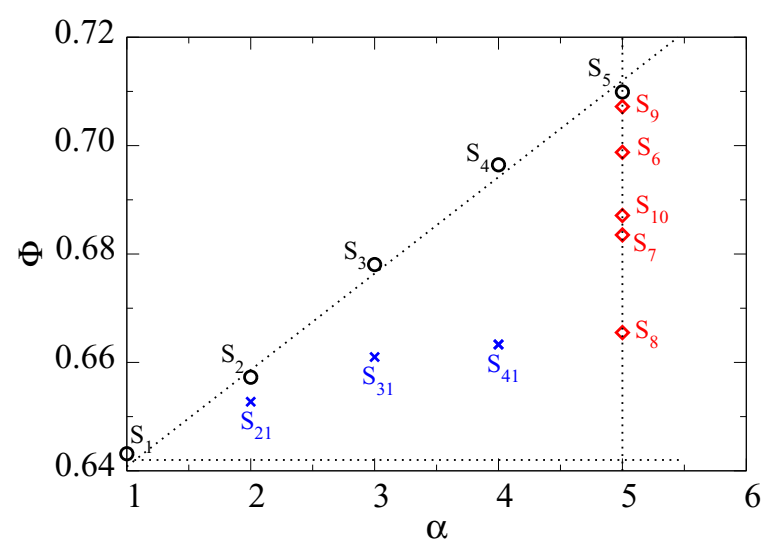

FIG. 4. Packing fraction $\Phi$ as a function of the size ratio $\alpha$ for all samples. The dotted lines are approximate borders of the accessible values of the packing fraction.

with other measured values of the RCP packing fraction in the monodisperse case $(\alpha=1)$, we get $\Phi=\Phi_{c} \simeq 0.643$ $[8,9,25,26]$. As in 2D simulations of disk packings [20], the samples (S2-S5) with a uniform distribution by volume fractions $(a=b=1)$, have the highest values $\Phi=\Phi_{v}(\alpha)$, which increase almost linearly with $\alpha$. For $\alpha=5, \Phi_{v}$ is as large as 0.710 . Interestingly, the linear fit passes also through the point $S 1\left(\alpha=1, \Phi=\Phi_{c}\right)$, corresponding to the monodisperse packing.

By contrast, the samples S21, S31, S41, and S8 with a uniform distribution in particle diameters $(a=3, b=1)$ have low packing fractions, increasing from $\Phi_{c}=0.643$ for $\alpha=1$ to 0.665 for $\alpha=5$. The size distribution in these samples (the same number of particles in all size classes) is such that small particles are too deficient in volume to fill efficiently the pore space between larger particles, and this leads to high porosity. Between these two limits, we see that for $\alpha=5$ the packing fraction increases substantially when $a$ declines (tending to the packing fraction for a uniform distribution by particle fractions) and increases when $b$ declines (tending to the packing fraction of uniform distribution by particle diameters). We expect even lower values of $\Phi$ as compared to that of uniform distributions by particle diameters if $a$ is further increased (beyond $a=3$ ). But obviously, the resulting packing fractions cannot be below $\Phi_{c}$. Hence, the lower limit in Fig. 4 is a horizontal line for which all samples have a packing fraction practically equal to $\Phi_{c}$. By increasing $b, \Phi$ will increase to reach a value $\Phi_{v}(\alpha)$ depending on $\alpha$ at the higher limit line.

Hence, in the space $(\alpha, \Phi)$, we can define three border lines:

(1) the high- $\Phi$ line, defined by the function $\Phi=\Phi_{v}(\alpha)$ and joining all data points with $a=b=1$,

(2) the low- $\Phi$ line, defined by $\Phi=\Phi_{c}$, and

(3) the vertical line $\alpha=5$ (in Fig. 4).

These lines are sketched in Fig. 4. The packing fraction varies for any given value of $\alpha$ between the two limits $\Phi_{c}$ and $\Phi_{v}(\alpha)$ as a function of $a$ and $b$. We conjecture that for the $\beta$ distribution of particle sizes, the packing fraction will always belong to the triangular space defined by the above three lines. The samples S21, S31, and S41 are inside this space. The 
variability of $\Phi$, and thus the effect of shape parameters on the packing fraction, increases with $\alpha$. Note that the linear relationship $\Phi_{v}(\alpha)$ is observed here only for the investigated values of $\alpha$. Obviously, it cannot be extended to larger values of $\alpha$ as the packing fraction cannot exceed 1 .

In real materials, the range of particle sizes can be broad, but all sizes are not statistically well represented. Moreover, for space-filling properties such as the packing fraction, the relevant parameter is not the number of particles but the total volume of particles of a given size. Many particles of small size can fill the same volume as do a few particles of large size. However, the number of particles is important for larger particles. A few large particles in a sample should not be considered on the same basis as size classes which are well represented in number and volume. Hence, to deal with a sample, it is easier to work with the quantiles of the volume distribution. As the largest particle size is not well defined (this is a single particle), we need to consider a size interval for which we have a large number of particles. In the same way, for small particles, we need to consider an interval for which we have a significant volume.

It is common in soil mechanics to consider the small-size interval as the interval representing $10 \%$ of the total volume. A particle diameter $d_{10}$ is defined as the quantile of this interval, i.e., the diameter such that the total volume of all smaller particles $\left(d<d_{10}\right)$ represents $10 \%$ of the total volume. In this sense, $d_{10}$ can be considered as the (conventional) size of the smallest class of particles. Statistically, it is more relevant than the size of very small particles that can be found in the sample and which are not significant in volume or number. The choice of $10 \%$ as the first limit is arbitrary and may be changed for unusual size distributions. In a similar construction, the largesize interval is the cumulative volume of particles, starting from the largest particle down to particles of a size $d_{60}$, such that the total volume of larger particles $\left(d>d_{60}\right)$ is $40 \%$ of the total volume. In other words, the total volume of particles of size $d<d_{60}$ represents $60 \%$ of the total volume. The choice of $40 \%$ for the volume fraction of the large-size interval (instead of $10 \%$ for small particles) is a way of ensuring that the number of particles in this interval is large. Hence, $d_{60}$ may be considered as the (conventional) size of the class of largest particles. Formally, from the definition of $h(d)$ in Eq. (3), the two quantiles defined above are given by

$$
\begin{aligned}
& d_{10}=h^{-1}(0.1) \\
& d_{60}=h^{-1}(0.6) .
\end{aligned}
$$

In soil mechanics, the CVD is characterized by the coefficient of uniformity $C_{u}$, defined as

$$
C_{u}=\frac{d_{60}}{d_{10}} .
$$

The "uniformity" refers to particle volume fractions. This coefficient measures in fact the degree of uniformity of the distribution in particle volumes. Depending on the shape of the distribution, $d_{60}$ can be close to $d_{10}$. This is the case when the amount of small particles is not enough to add up to $10 \%$ of the total volume unless extended to particle sizes close to $d_{60}$. This is also the case when the number of particles larger than $d_{10}$ or their volumes is enough to reach
TABLE III. Coefficient of uniformity $C_{u}$ of the samples.

\begin{tabular}{lccccccc}
\hline \hline Sample & $\mathrm{S} 1$ & $\mathrm{~S} 2$ & $\mathrm{~S} 3$ & $\mathrm{~S} 4$ & $\mathrm{~S} 5$ & $\mathrm{~S} 6$ & $\mathrm{~S} 7$ \\
$C_{u}$ & 1.00 & 1.45 & 1.83 & 2.15 & 2.43 & 2.12 & 1.88 \\
\hline Sample & $\mathrm{S} 8$ & $\mathrm{~S} 9$ & $\mathrm{~S} 10$ & $\mathrm{~S} 21$ & $\mathrm{~S} 31$ & $\mathrm{~S} 41$ & \\
$C_{u}$ & 1.58 & 2.61 & 1.90 & 1.41 & 1.53 & 1.55 & \\
\hline \hline
\end{tabular}

$60 \%$ of the total volume for a value of $d_{60}$ close to $d_{10}$. In these cases, $C_{u}$ is close to $1 . C_{u}$ increases, by definition, when the intermediate populations of particles are such that the volumes are evenly distributed. Considering that $d_{10}$ and $d_{60}$ are effective diameters of the smallest and largest particles in the packing (instead of $d_{\min }$ and $d_{\max }$ as the discrete extreme values), $C_{u}$ can be thought of as an effective size span of the distribution, and it is expected to play the same role as $\alpha$ for arbitrary distributions, including those for which the extreme particle sizes are statistically ill defined. This is precisely the advantage of using $C_{u}$ as a descriptor of CVD.

Let us calculate $C_{u}$ for the two uniform distributions respectively in terms of volume fractions and particle diameters. The uniform CVD in volume fractions is given by

$$
h(d)=\frac{d-d_{\min }}{d_{\max }-d_{\min }}=d_{r} .
$$

Using Eq. (7), we get

$$
\begin{aligned}
& d_{10}=0.1\left(d_{\max }-d_{\min }\right)+d_{\min } \\
& d_{60}=0.6\left(d_{\max }-d_{\min }\right)+d_{\min }
\end{aligned}
$$

so that

$$
C_{u}=\frac{0.6(\alpha-1)+1}{0.1(\alpha-1)+1} .
$$

This equation implies that $C_{u}$ does not depend only on the shape parameters but increases also with the size ratio $\alpha$. For a uniform distribution by volume fractions, $C_{u}$ tends asymptotically to 6 with increasing value of $\alpha$.

In the uniform distribution in particle diameters, $P(d)$ is independent of $d$. From Eq. (3), given that $V(d)=\pi d^{3} / 6$, we get

$$
h(d)=\frac{d^{4}-d_{\min }^{4}}{d_{\max }^{4}-d_{\min }^{4}}
$$

which yields the following expression for the coefficient of uniformity:

$$
C_{u}=\left\{\frac{0.6\left(\alpha^{4}-1\right)+1}{0.1\left(\alpha^{4}-1\right)+1}\right\}^{1 / 4} .
$$

The asymptotic value as $\alpha \rightarrow \infty$ is $6^{1 / 4} \simeq 1.565$, which is the highest value of the coefficient of uniformity for a uniform distribution by particle diameters. The values of $C_{u}$ for all our samples are given in Table III.

Note that the uniform distributions by particle diameters and volumes can be extended to a general power-law 


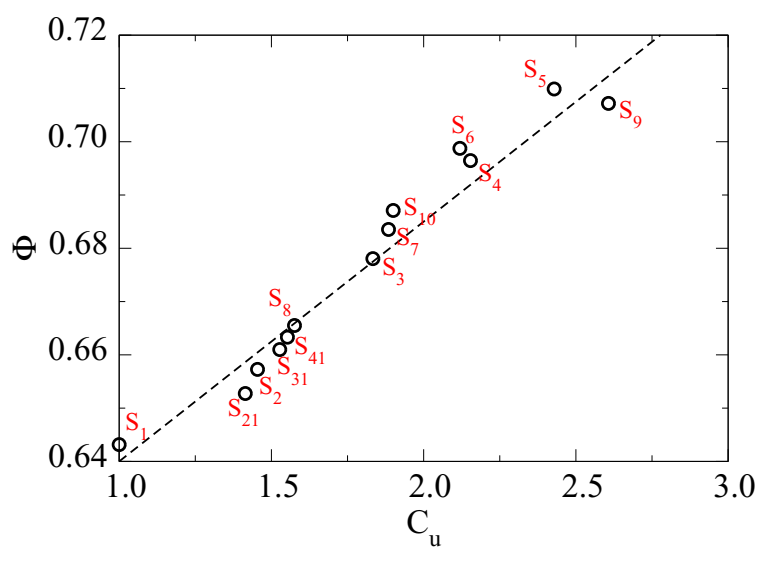

FIG. 5. Packing fraction $\Phi$ as a function of the coefficient of uniformity $C_{u}$ for all samples.

CVD,

$$
h(d)=\frac{d^{q}-d_{\min }^{q}}{d_{\max }^{q}-d_{\min }^{q}},
$$

with exponent $q$ and a coefficient of uniformity given by

$$
C_{u}=\left\{\frac{0.6\left(\alpha^{q}-1\right)+1}{0.1\left(\alpha^{q}-1\right)+1}\right\}^{1 / q} .
$$

Hence, the asymptotic value of $C_{u}$ varies as $6^{1 / q}$ and tends to 1 with increasing $q$.

Figure 5 shows the packing fraction as a function of the coefficient of uniformity for all our samples. We observe a strong correlation between $C_{u}$ and $\Phi$. This is quite remarkable given the dispersion of the values of $\Phi$ observed in Fig. 4 as a function of $\alpha$. This means that, independently of the values of the three parameters of the distribution up to small fluctuations, the packing fraction is a roughly linear function of $C_{u}$. This correlation is consistent with the interpretation of $C_{u}$ as an effective size span that accounts for both the extent of particle sizes and the shape of their size distribution. It implies that particle size distributions with higher value of $C_{u}$ can assemble into higher values of the packing fraction. The difference between the two uniform distributions by particle volumes and particle diameters can thus be attributed to the systematically very different values of their respective coefficients of uniformity. Below, we consider other attributes of the isostatic packings, such as connectivity and force transmission, and analyze their dependence on the CVD parameters and coefficient of uniformity.

\section{PACKING CONNECTIVITY}

The contact network can be analyzed using various mathematical descriptors. We consider here the connectivity of the particles defined as the proportions $P_{d c}\left(d_{r}, c\right)$ of particles of reduced size $d_{r}$ and having exactly $c$ contacts. The connectivity of particles independently of their sizes is $P_{c}=$ $\sum_{d_{r}} P_{d c}\left(d_{r}, c\right)$, which is the proportion of particles with $c$ contacts. The coordination number is given by $Z=\sum_{c} c P_{c}$. Figure 6 shows $Z$ as a function of $C_{u}$ for all samples. We see that $Z$ declines slightly as $C_{u}$ increases, and all the data

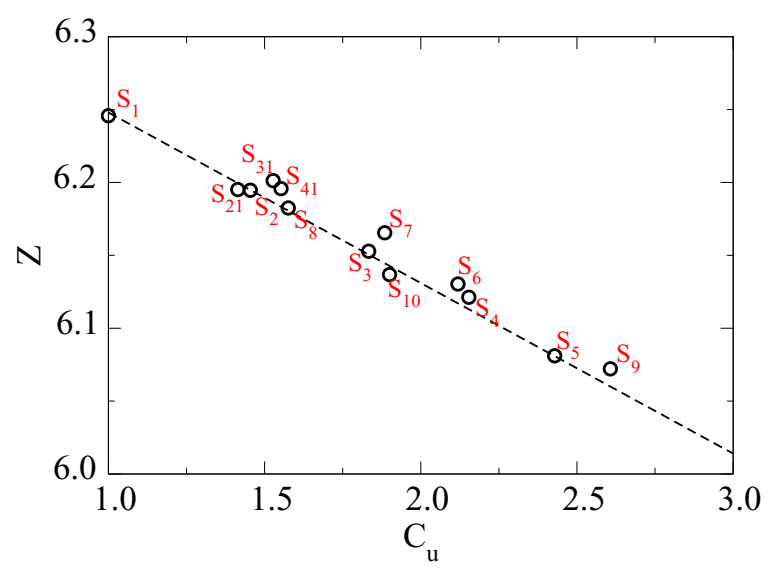

FIG. 6. Coordination number $Z$ as a function of the coefficient of uniformity $C_{u}$ for all samples.

points are, up to small variability, well aligned on a single nearly linear curve. These values of $Z$ above 6 indicate that the packing is overconstrained as a result of small elastic deflections at the contact points between particles. The decrease of $Z$ toward 6 as $C_{u}$ increases is consistent with the fact that for a fixed confining stress $\sigma_{z z}$, the average contact force, and hence the average elastic deflection, declines as polydispersity increases.

Figure 7 shows $P_{c}$ for different values of $c . P_{c}$ is negligibly small for $c \leqslant 3$. Indeed, for $c=3$, the force balance is possible only if the three normal force vectors are on the same plane, which is quite improbable. In the same way, force balance for $c=2$ implies equal forces on diametrically opposite contact points, which is a metastable configuration of practically no chance to occur without friction. Finally, for $c=1$ the contact force is necessarily zero. In the monodisperse case $\left(C_{u}=1\right)$, we observe a peak value for $c=6$, which is consistent with $Z \simeq 6$, i.e., the values are distributed around the peak value. For $C_{u}=1.41$ and $C_{u}=1.45$ (both corresponding to $\alpha=2$ ), the peak occurs at $c=5$. For all larger values of $C_{u}, P_{c}$ is peaked on $c=4$ with a value that increases with $C_{u}$. For $C_{u}=2.43$, nearly $45 \%$ of particles have four contacts whereas only $12 \%$ of particles have six contacts.

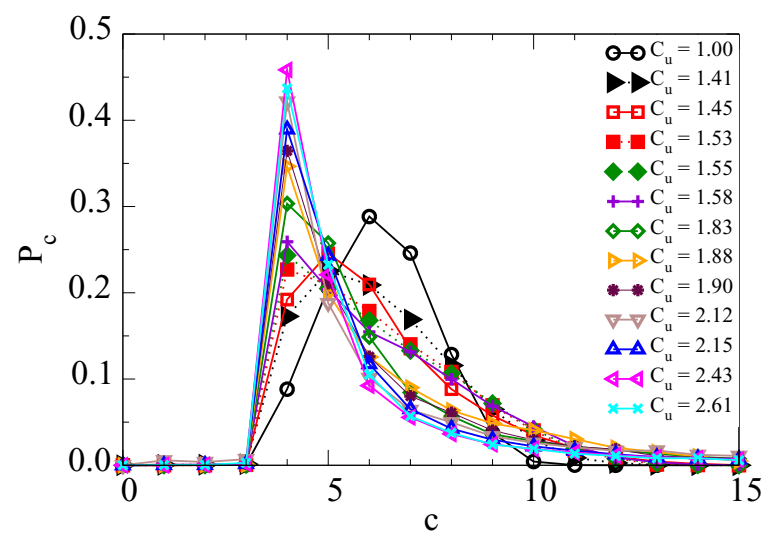

FIG. 7. The fraction $P_{c}$ of particles with $c$ contacts for different values of $C_{u}$. 


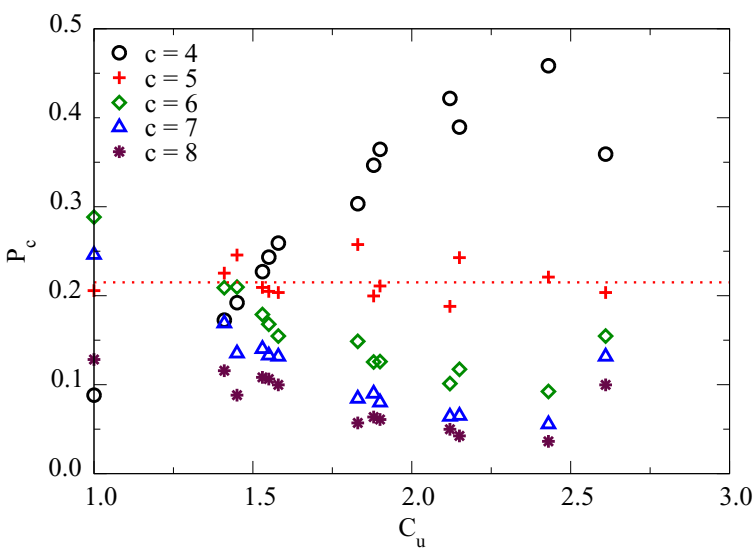

FIG. 8. The fraction $P_{c}$ of particles with $c$ contacts as a function $C_{u}$ for different values of $c$.

The same data are shown in Fig. 8 by plotting $P_{c}$ as a function of $C_{u}$ for different values of $c$. We see that $P_{4}$ is the only class that increases with $C_{u}$, whereas $P_{5}$ is nearly independent of $C_{u}$. For all other values of $c, P_{c}$ declines as $C_{u}$ increases. The large value of $P_{4}$ indicates that the configurations in which a particle is equilibrated by four neighboring contact particles are more stable than other configurations. They can be considered as minimally locally jammed particles [5]. Since the peak value increases with $C_{u}$, it is expected that all such particles are of small size. This is indeed the case, as seen in Fig. 9 where we have plotted the distributions $P_{d c}$ and $P_{d c} / P_{c}$ as a function of $d_{r}$ for different values of $c$ in the case $C_{u}=2.43$. We see that $75 \%$ of particles with $c=4$ contacts (corresponding to $\simeq 34 \%$ of all particles) are in the class of the smallest particles $\left(d_{r}=0\right)$. Almost $40 \%$ of particles with each value of $c$ belong to a specific size class. In Fig. 9(b), these are the peaks shifting to the larger values of $d_{r}$ as $c$ increases. The remaining $50 \%$ are distributed in the neighboring size classes. The particles with $c=4$ have a much higher peak value in the size class $d_{r}=0$ and only $25 \%$ of them belong to the classes of larger particle sizes.

The above picture of particle connectivities and the effect of PSD can be pushed farther by considering the contact anisotropies $a_{c}$ of the samples. The anisotropy is generally calculated from the second-order fabric tensor $F_{\alpha \beta}$ defined by

$$
F_{\alpha \beta}=\frac{1}{N_{c}} \sum_{i} n_{\alpha}^{i} n_{\beta}^{i}=\left\langle n_{\alpha} n_{\beta}\right\rangle,
$$

where $n_{\alpha}^{i}$ is the $\alpha$ component of the unit normal vector $\vec{n}^{i}$ of the contact $i$ and $N_{c}$ is the number of contacts. By definition, we have $\operatorname{tr}(F)=1$, and thus the anisotropy of the contact orientations can be characterized by the differences $F_{1}-F_{2}$, $F_{2}-F_{3}$, and $F_{1}-F_{3}$ between the principal values $F_{1} \geqslant F_{2} \geqslant$ $F_{3}$ of the fabric tensor. Here, we take the largest difference:

$$
a_{c}=F_{1}-F_{3} \text {. }
$$

The major principal direction of $F$ represents the privileged orientation of contacts.

Due to the absence of friction between particles, we expect a very low anisotropy of the samples, which builds up only if stable chains of contacts can be formed along the compaction

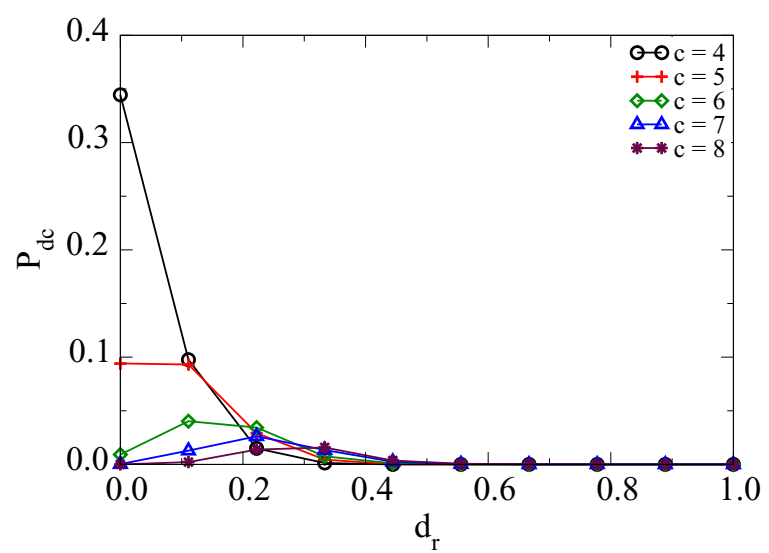

(a)

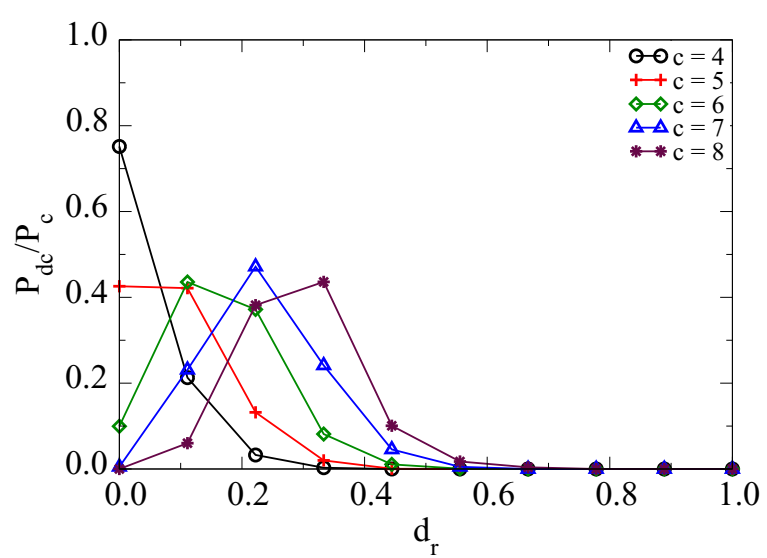

(b)

FIG. 9. (a) The connectivity $P_{d c}$ as a function of reduced particle diameter $d_{r}$ and different values of $c$ for $C_{u}=2.43$, and (b) the same data normalized by the fraction $P_{c}$ of particles with $c$ contacts (b).

axis. Figure 10 shows the calculated anisotropies of all our samples as a function of $C_{u}$. We see that the fabric anisotropy is quite weak in all samples. It increases from 0.005 in the monodisperse sample to 0.025 in the samples S5 and S9.

The same definition of the fabric tensor can be applied to each particle by restricting the summation in Eq. (16) to its

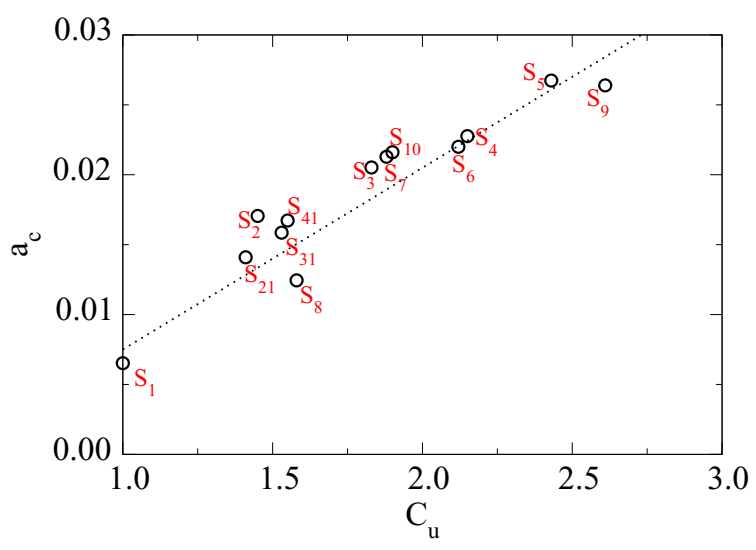

FIG. 10. Overall anisotropy $a_{c}$ of the samples as a function of their coefficient of uniformity $c_{u}$. 


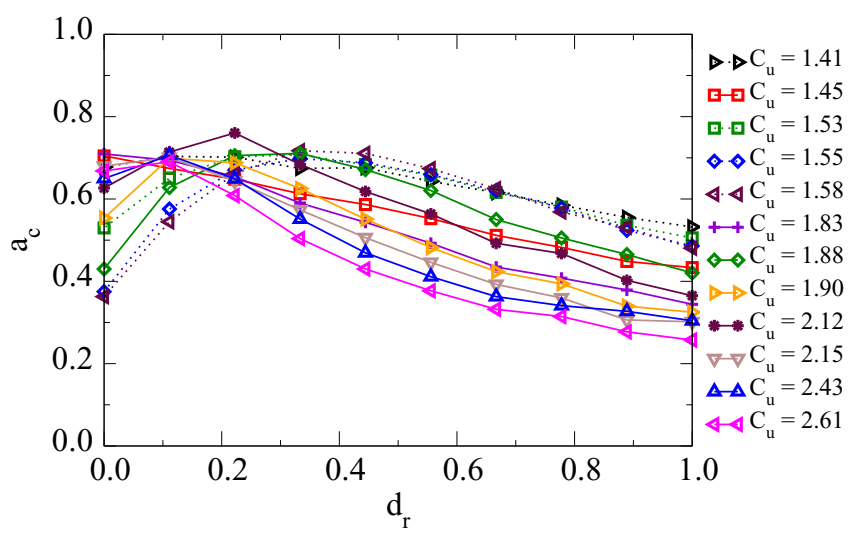

FIG. 11. Particle contact anisotropy $a_{c}$ as a function of reduced particle size $d_{r}$ for all samples with different values of the coefficient of uniformity.

contacts. The average anisotropy of particles is displayed in Fig. 11 as a function of reduced particle size $d_{r}$ for different values of $C_{u}$. Here we find far larger values of the anisotropy than the overall anisotropies. The particle anisotropies can be large, but they do not sum up to large values as a result of the random orientations of particle fabric tensors. For those values of $C_{u}$ corresponding to uniform size distributions by volume fractions, $a_{c}$ decreases as $d_{r}$ is increases. This decrease is a consequence of the increase in the number of contacts of particles of increasing size. Indeed, the larger number of contacts has a randomizing effect on the contact orientations, leading to lower anisotropy [27]. For those values of $C_{u}$ corresponding to the PSD with a low number of small particles (such as uniform distributions by particle size), $a_{c}$ increases before decreasing again as $d_{r}$ tends to 1 . This behavior reflects the role of small particles, as discussed previously. For example, the small $P_{4}$ particles have a low anisotropy as their contact neighbors have larger diameters and hence well-distributed orientations as a result of their steric exclusions. The peak value of anisotropy occurs in the range $0.2<d_{r}<0.3$.

\section{STRESS TRANSMISSION}

A feature worth investigating in polydisperse RCP packings is the force transmission. As the porosity declines with increasing size polydispersity, one might expect that the forces get more homogeneously distributed [28]. Instead, we observe that the force distributions are increasingly more inhomogeneous when $C_{u}$ increases, as shown in Fig. 12. The probability distribution function (PDF) of normal forces $f$ has a marked exponential shape in the range of forces above the mean force $\langle f\rangle$. Its width increases with $C_{u}$, and at the same time, the proportion of weak forces (below the mean) increases. The force peak centered on $f \simeq 0.5\langle f\rangle$, disappears for $C_{u}>1.53$. This enhanced force inhomogeneity can be attributed to the higher topological inhomogeneity of the contact network, as suggested by the increasingly broader shape of $P_{c}$ (Fig. 8) with increasing $C_{u}$.

Figure 13 displays the contact force networks for $C_{u}=$ 2.43 (S5) and $C_{u}=1.58$ (S8). It is seen that, although the coordination number and size polydispersity are nearly the

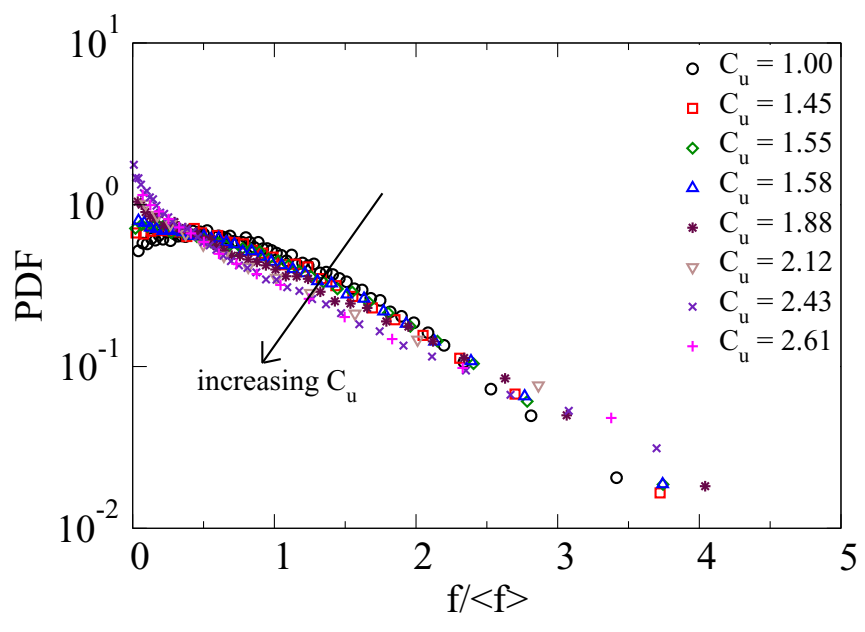

FIG. 12. Probability density function of normal contact forces $f$ normalized by the mean force $\langle f\rangle$ for different gradation $C_{u}$.

same in both samples, the force structures are quite different. In S5 (uniform distribution by volume fractions) there are many more small forces, and the network has a hierarchical structure, whereas in S8 (uniform distribution by particle diameters) the network is similar to that in monodisperse packings due to the presence of larger voids between large particles, only partially filled by smaller particles.

It is interesting to see how the forces are distributed among different size classes. The interclass forces $f_{i c}(\alpha, \beta)$ are the

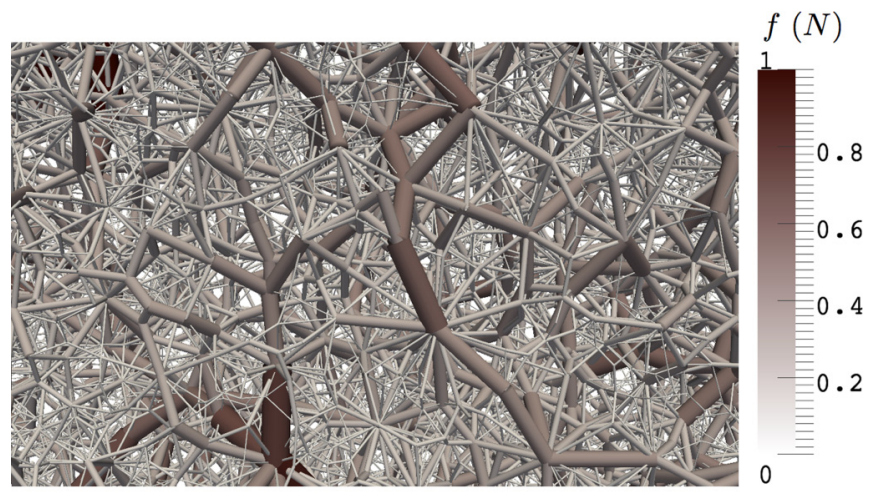

(a)

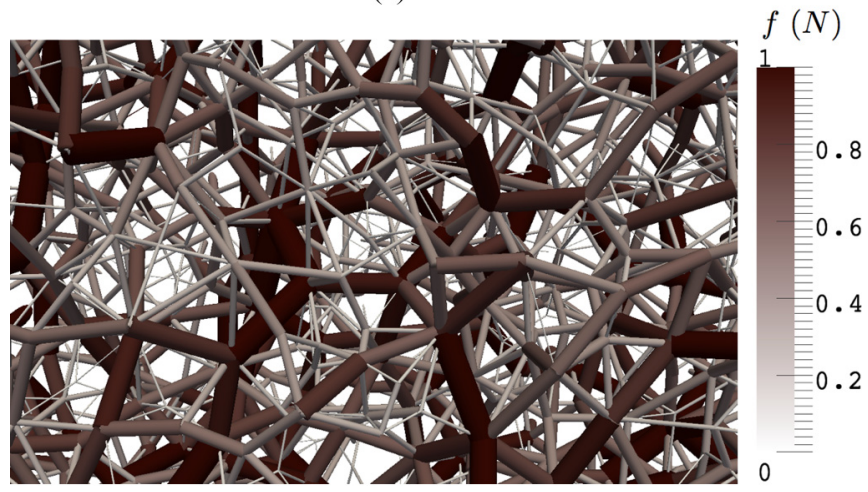

(b)

FIG. 13. Contact force networks for $C_{u}=2.43$ (a) and $C_{u}=1.58$ (b). Line thickness and color are proportional to contact force. 


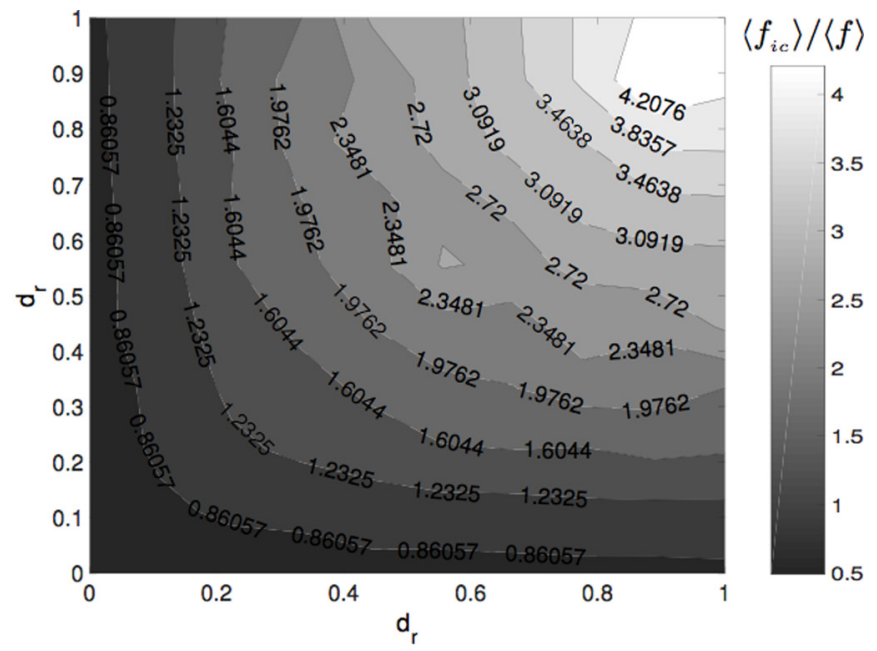

(a)

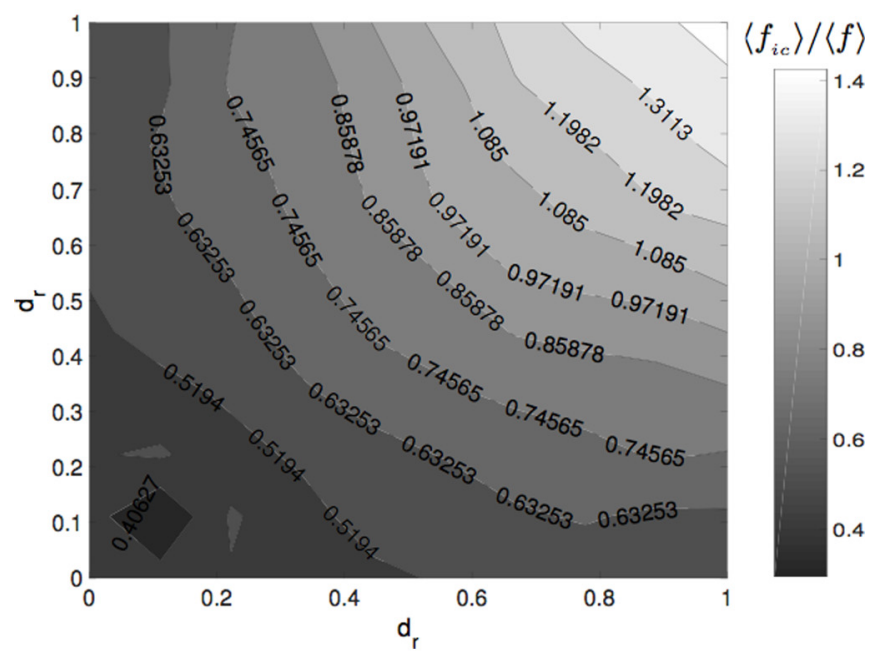

(b)

FIG. 14. Multiclass gray-level map of the mean interclass forces $\left\langle f_{i c}\right\rangle$ normalized by mean packing force $\langle f\rangle$ for the samples $\mathrm{S} 5\left(C_{u}=\right.$ 2.43) (a) and S8 ( $\left.C_{u}=1.58\right)$ (b).

forces between two particles belonging to the classes of reduced sizes $d_{r}=\alpha$ and $d_{r}=\beta$, respectively. We consider its average value $\left\langle f_{i c}\right\rangle(\alpha, \beta)$ for all such particles. Figure 14 shows a gray-level map of $\left\langle f_{i c}\right\rangle$ normalized by the mean force $\langle f\rangle$ for two samples. For a particle of a given size (belonging to a size class), $\left\langle f_{i c}\right\rangle$ increases with the size of its partner. Its largest value occurs when two particles are in the largest class. This clearly shows that the strong force chains are captured by the larger particles. This trend is much more pronounced for packings of higher $C_{u}$. In Fig. 15 we have plotted $\left\langle f_{i c}\right\rangle\left(d_{r}, d_{r}\right)$ for $d_{r}=0,0.5$, and 1 . The mean force $\left\langle f_{i c}\right\rangle$ in the smallest class is practically independent of $C_{u}$ with a value $\simeq 0.5\langle f\rangle$. In contrast, in the two other classes of larger and largest particles sizes, $\left\langle f_{i c}\right\rangle$ increases almost linearly with $C_{u}$ up to fluctuations that reflect the CVD parameters.

The contact forces can also be used to calculate the average pressures acting on the particles $\sigma_{p}$ (the sum of normal forces on each particle divided by its surface area). The mean value of particle pressures in each size class $\left\langle\sigma_{p}\right\rangle$ are shown in

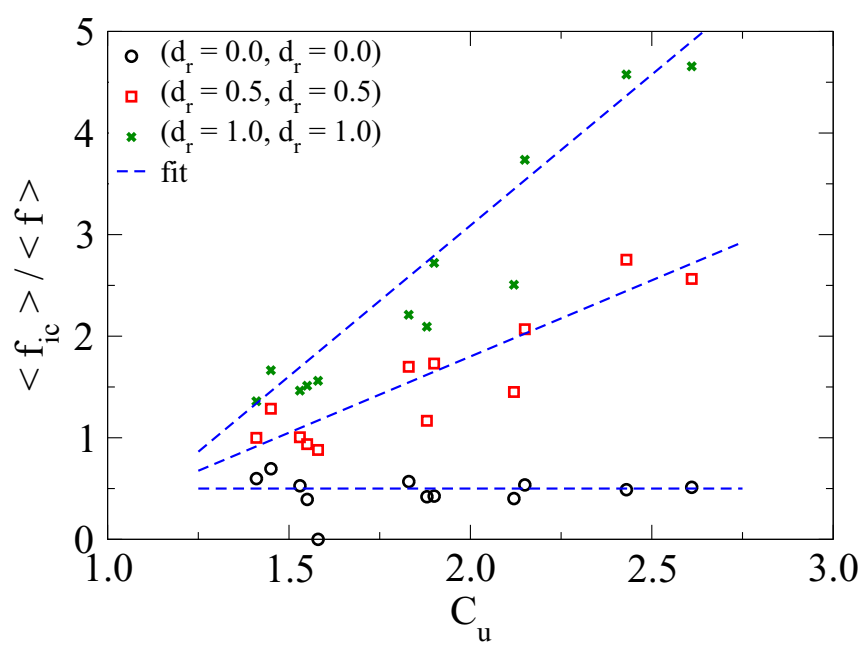

FIG. 15. Mean interclass force $\left\langle f_{i c}\right\rangle\left(d_{r}, d_{r}\right)$ normalized by the mean force for $d_{r}=0,0.5$ and 1 , as a function of the coefficient of uniformity $C_{u}$.

Fig. 16 as a function of reduced size $d_{r}$ for several values of $C_{u}$. The pressures are normalized by the mean pressure $\langle\sigma\rangle$ in each sample. We see that, except for S5 and $\mathrm{S} 9,\left\langle\sigma_{p}\right\rangle /\langle\sigma\rangle$ is an increasing function of $d_{r}$. Hence, not only the average force in each class but also the average pressure increase with particle size. The case of the samples S5 and S9 is, however, significant since these samples differ from other samples in their larger proportion of smaller size particles. In these samples, up to statistical fluctuations, the particle pressure is independent of particle size. In other words, the small particles take more active part in stress transmission than in other samples. These samples are also those that have the highest packing fraction. Figure 17 shows a snapshot of particles with a color-level representation of their pressures in the samples S5 and S8. In S5, the large particles have mostly high pressures, whereas the small particles carry both low

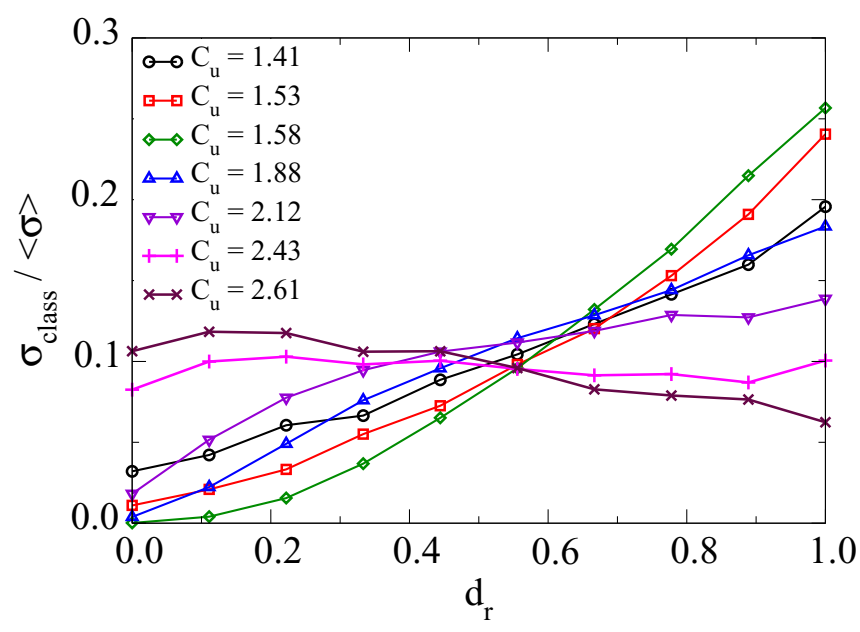

FIG. 16. Normalized mean class pressure $\sigma_{p}$ as a function of reduced diameter $d_{r}$ for different values of $C_{u}$. The pressures are normalized by the mean pressure $\sigma$ in each sample. 


$$
\sigma_{p} / \sigma_{0}
$$

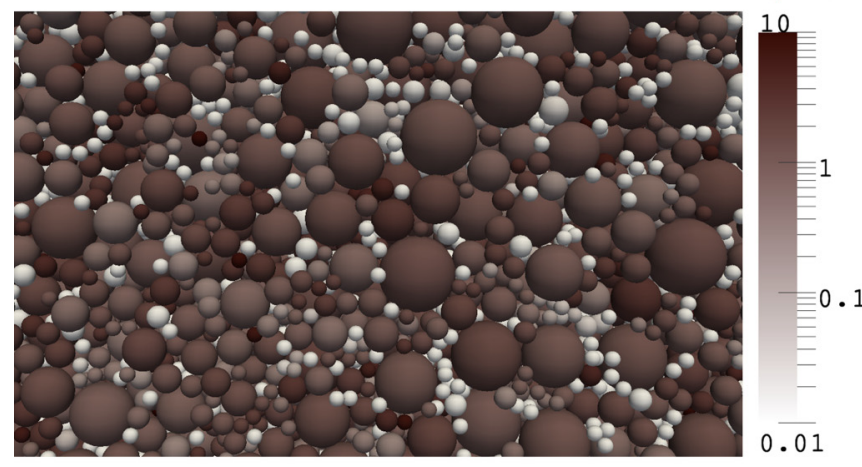

(a)

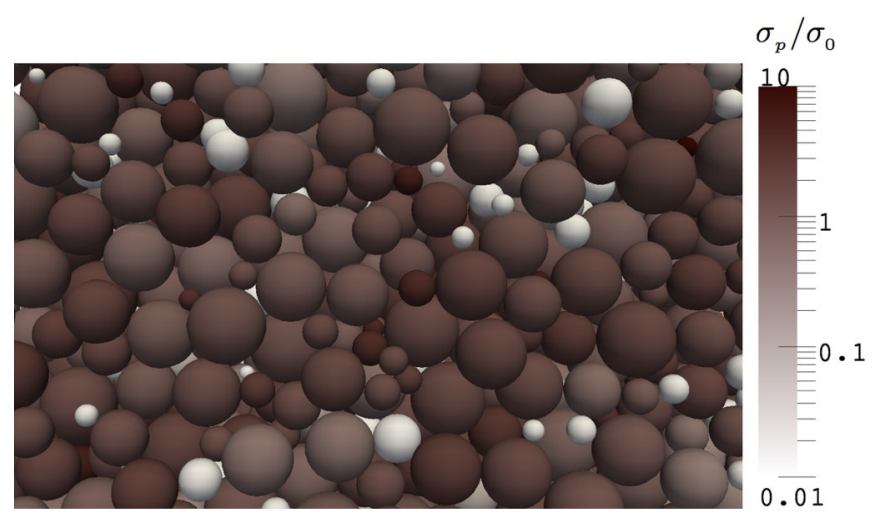

(b)

FIG. 17. Snapshots of samples with particles colored according to applied total stress $\sigma_{p}$ on each particle normalized by the mean packing stress $\sigma_{0}$ both figures for $\alpha=5$. (a) Packing S5 with $C_{u}=$ 2.43 and (b) packing S8 with $C_{u}=1.58$.

and high stresses depending on their position in the contact network. In S8, the large particles carry most of the stress.

\section{CONCLUSION}

In this paper, we used DEM simulations to build polydisperse RCP packings of spherical particles by setting the interparticle friction coefficient to zero. The cumulate volume distributions of the particles were generated according to the incomplete $\beta$ function with three parameters: A size span and two shape parameters. The number of particles and size classes were carefully determined to impose a minimum number of particles in the largest-size class and a minimum total volume of the particles in the smallest-size class. The equilibrium state reached after a long relaxation under load represents the highest packing fraction for each size distribution.

A central finding of this work is that, despite different size spans and shapes, the coefficient of uniformity plays the role of a first-order parameter that scales correctly the packing fraction, particle connectivity, and stress transmission. To small variations, the packing fraction, the proportion of particles with four contacts and mean forces between or inside different size classes are increasing functions of the coefficient of uniformity. The coefficient of uniformity may be viewed as an effective size ratio that takes into account the particle volumes. Hence, it can bring together very different data such as those from the uniform size distributions by volume fractions and diameters of the particles.

The stress transmission properties reveal the important effects of the PSD shape more specifically in connection with the class of the smallest particles. These particles may be more or less in number depending on the PSD shape. For the shapes in excess of small particles (as compared to the uniform distribution by volume fractions), the mean force per size class is nearly independent of particle size. The active role of small particles in stress transmission depends thus on their number (and distribution in space, which is quite homogeneous in our simulations). For other distributions, in lack of small particles, the mean interclass force increases with particle size. We also underlined the increasing inhomogeneity of force transmission, reflected in the increasing width of force PDFs, as the coefficient of uniformity increases. This feature is counterintuitive in the sense that the space is more homogeneously filled by the particles as the coefficient of uniformity increases. In fact, force transmission is rather strongly correlated with particle connectivity, which becomes more complex and inhomogeneous as the coefficient of uniformity increases. Its signature appears also in local particle environments such as particle pressures and anisotropies.

The packing fraction, microstructure and force transmission properties investigated in this paper concern RCP packings prepared by setting the interparticle friction coefficient to zero. For this reason, they cannot be generalized to sphere packings prepared with a nonzero friction coefficient. Nevertheless, on some grounds such as the prevailing effect of particle size distribution compared to dilatancy or anisotropy induced by deformations, we expect similar trends as a function of the coefficient of uniformity in steady shear and under isotropic compaction with nonzero friction [21]. The RCP configurations that we analyzed in this paper thus can be used as initial states for long shear tests with different values of the friction coefficient. We are also interested in the effects of other size distributions than those considered in this paper with the idea of further consolidating our results.

\section{ACKNOWLEDGMENTS}

The authors acknowledge the support of MIT Energy Initiative, Natural Sciences and Engineering Research Council of Canada (NSERC), ICoME2 Labex (ANR-11-LABX-0053), and the A*MIDEX projects (ANR-11-IDEX-0001-02) cofunded by the French program "Investissements d'Avenir," managed by the French National Research Agency (Agence Nationale de la Recherche).
[1] T. Aste and D. Weaire, The Pursuit of Perfect Packing (Institute of Physics Publishing, Philadelphia, 2000).
[2] S. Torquato and F. H. Stillinger, Rev. Mod. Phys. 82, 2633 (2010). 
[3] F. de Larrard, Concrete Mixture Proportioning (E \& FN Spon, London, New York, 1999).

[4] P. S. Dodds and J. S. Weitz, Phys. Rev. E 65, 056108 (2002).

[5] A. Donev, S. Torquato, F. H. Stillinger, and R. Connelly, J. Appl. Phys. 95, 989 (2004).

[6] J. G. Berryman, Phys. Rev. A 27, 1053 (1983).

[7] S. Torquato, T. M. Truskett, and P. G. Debenedetti, Phys. Rev. Lett. 84, 2064 (2000).

[8] P.-E. Peyneau and J.-N. Roux, Phys. Rev. E 78, 041307 (2008).

[9] J.-N. Roux, Phys. Rev. E 61, 6802 (2000).

[10] H. J. Herrmann, R. Mahmoodi Baram, and M. Wackenhut, Physica A 330, 77 (2003).

[11] O. U. Uche, F. H. Stillinger, and S. Torquato, Physica A 342, 428 (2004).

[12] A. Donev, F. H. Stillinger, and S. Torquato, Phys. Rev. Lett. 96, 225502 (2006).

[13] C. Voivret, F. Radjaï, J.-Y. Delenne, and M. S. El Youssoufi, Phys. Rev. Lett. 102, 178001 (2009).

[14] M. Ammi, D. Bideau, and J. P. Troadec, J. Phys. D: Appl. Phys. 20, 424 (1987).

[15] A. Gervois and D. Bideau, in Disorder and Granular Media, edited by D. Bideau (Elsevier, Amsterdam, 1993).
[16] T. Aste, Phys. Rev. E 53, 2571 (1996).

[17] R. Jullien, P. Jund, D. Caprion, and D. Quitmann, Phys. Rev. E 54, 6035 (1996).

[18] R. Jullien and P. Meakin, Colloids Surf. A 165, 405 (2000).

[19] A. R. Kansal, T. M. Truskett, and S. Torquato, J. Chem. Phys. 113, 4844 (2000).

[20] C. Voivret, F. Radjai, J.-Y. Delenne, and M. S. El Youssoufi, Phys. Rev. E 76, 021301 (2007).

[21] D.-H. Nguyen, E. Azéma, F. Radjai, and P. Sornay, Phys. Rev. E 90, 012202 (2014).

[22] D. Cantor, E. Azéma, P. Sornay, and F. Radjai, Phys. Rev. E 98, 052910 (2018).

[23] N. Estrada, Phys. Rev. E 94, 062903 (2016).

[24] J. K. Mitchell and K. Soga, Fundamentals of Soil Behavior, 2nd ed. (Wiley, New York, 2005).

[25] W. S. Jodrey and E. M. Tory, Phys. Rev. A 32, 2347 (1985)

[26] P.-E. Peyneau and J.-N. Roux, Phys. Rev. E 78, 011307 (2008).

[27] F. Radjai, J.-Y. Delenne, É. Azema, and S. Roux, Granul. Matter 14, 259 (2012).

[28] H. Laubie, F. Radjai, R. Pellenq, and F.-J. Ulm, Phys. Rev. Lett. 119, 075501 (2017). 